\title{
Steiner Forests on Stochastic Metric Graphs
}

\author{
Vangelis Th. Paschos* \\ paschos@lamsade.dauphine.fr \\ LAMSADE, CNRS UMR 7024 and Université Paris-Dauphine, France \\ Orestis A. Telelis* \\ telelis@di.uoa.gr \\ Department of Informatics and Telecommunications, University of Athens, Greece \\ Vassilis Zissimopoulos* \\ vassilis@di.uoa.gr \\ Department of Informatics and Telecommunications, University of Athens, Greece
}

\begin{abstract}
We consider the problem of connecting given vertex pairs over a stochastic metric graph, each vertex of which has a probability of presence independently of all other vertices. Vertex pairs requiring connection are always present with probability 1 . Our objective is to satisfy the connectivity requirements for every possibly materializable subgraph of the given metric graph, so as to optimize the expected total cost of edges used. This is a natural problem model for cost-efficient Steiner Forests on stochastic metric graphs, where uncertain availability of intermediate nodes requires fast adjustments of traffic forwarding. For this problem we allow a priori design decisions to be taken, that can be modified efficiently when an actual subgraph of the input graph materializes. We design a fast (almost linear time in the number of vertices) modification algorithm whose outcome we analyze probabilistically, and show that depending on the a priori decisions this algorithm yields 2 or 4 approximation factors of the optimum expected cost. We also show that our analysis of the algorithm is tight.
\end{abstract}

\section{Introduction}

We consider the problem of laying out routes that connect simultaneously given sourcedestination vertex pairs over a metric graph $G_{0}\left(V_{0}, E_{0}\right)$. Vertices of the metric graph $G_{0}$ other than the sources and destinations may be used, but we are uncertain of their availability, in that each such vertex is present with some probability independently of all other vertices. Sources and destinations are present with probability 1 . Our objective is to take some a priori decisions regarding the layout of required routes, so as to be able to come up with feasible routes for every possibly materializable subgraph $G_{1}\left(V_{1}, E_{1}\right), V_{1} \subseteq V_{0}$, of $G_{0}$, and minimize the expected total cost of edges used over the distribution of all such subgraphs. This is the well known Steiner Forest problem, defined over a stochastic metric graph $G_{0}$.

A brute-force way to cope with this problem is to precompute a feasible and approximate (or maybe optimum) solution for every possible subgraph of $G_{0}$ that may materialize, and

\footnotetext{
*Authors partially supported by the Greek Ministry of Education and Research under the project PYTHAGORAS II
} 
apply an appropriate solution when the subgraph actually appears. In principle there need not be a constraint on the computational effort applied for taking a priori decisions, as long as they support fast response to the actually materialized data. In this light however, we require that such a response should be of strictly lower complexity compared to the a priori computational effort. A straightforward pattern for implementing this setting is for example to compute an optimum a priori solution over $G_{0}$, and if this solution is not feasible for the materialized subgraph $G_{1}$, use a polynomial-time approximation algorithm to obtain a completely different feasible solution for $G_{1}$. On the other hand, a natural challenge is to design such an efficient response strategy (algorithm), that can be supported by polynomialtime computable a priori decisions. In this paper we design and analyze such a strategy for repairing an a priori polynomial-time computable feasible solution for $G_{0}$, so as to render it feasible for $G_{1}$. We show that this strategy also approximates the optimum expected cost over all materializable subgraphs $G_{1}$.

The problem model we consider finds natural application in networks, where uncertain availability of intermediate nodes requires fast adjustments of traffic forwarding. The Steiner Forest problem is a well-known $N P$-hard multicommodity network design problem (even in metric graphs), generalizing the Steiner tree problem, and the only known approximation algorithm yields approximation factor 2 and was analyzed in [1, 8] (see also [18]). Recent years have seen a detailed study and sensitivity analysis of this algorithm, mainly in the context of Stochastic Network Design, which owes its roots to Stochastic Programming [5, 4], where some elements of the input data set to an optimization problem are associated to a distribution describing their probability of occurence. Stochastic Programming was introduced by the seminal work of Dantzig [5] and thereafter has evolved into an independent discipline of Operations Research that handles uncertainty in optimization problems by usage of probabilities, statistics and mathematical programming (see [4] for a description of the field). We refer the reader to [11, 7] for approximation results on Stochastic Steiner Forest models and to $[13,12,10,6,9]$ for additional recent approximation results on stochastic network design problems in general.

Our work is mostly related to the framework of Probabilistic Combinatorial Optimization, introduced in $[2,14]$, where repairing strategies as the one described previously are analyzed probabilistically, so that the expected cost of their outcome can be computed efficiently (this ensures that the problem of taking a priori decisions for a particular strategy belongs to class NPO). Several network design problems have been treated in the probabilistic combinatorial optimization framework, including minimum coloring [17], maximum independent set [16], longest path [15], and minimum spanning tree [3]. Apart from probabilistic analysis of repairing strategies, results in $[17,16]$ also include derivation of approximability properties.

The article is structured as follows. At first we introduce notation. In section 2 we present a repairing strategy (algorithm) and derive the expected cost of the repaired feasible solution for the actually materialized subgraph. Approximation properties of the proposed strategy with respect to a priori decisions (solutions) are analyzed in section 3. We show that our approximation results are tight in section 4 , and conclude in section 5 .

Notation In what follows we denote by $G_{0}\left(V_{0}, E_{0}\right)$ the input metric graph and let $\left\langle s_{r}, t_{r}\right\rangle$, $r=1 \ldots k$, denote the $k$ pairs that we have to connect for the Steiner Forest instance. Each vertex $v_{i} \in V_{0} \backslash\left\{s_{r}, t_{r} \mid r=1 \ldots k\right\}$ is accociated to a probability $p_{i}$ of survival in the actually materialized graph $G_{1}\left(V_{1}, E_{1}\right), V_{1} \subseteq V_{0}$. Vertices $s_{r}, t_{r}, r=1 \ldots k$ are assumed to be always 
present in $G_{1}$. $G_{1}$ emerges as the complete metric subgraph of $G_{0}$, by an independent random Bernoulli trial for each vertex $v_{i} \in V_{0}$.

We will elaborate on a feasible a priori solution that is a forest. We denote it as an edge subset $F_{0} \subseteq E_{0}$, consisting of $f_{0}$ trees and write $F_{0}=\cup_{l=1}^{f_{0}} T_{0, l}$. A feasible (possibly repaired) solution over the actually materialized subgraph $G_{1}$ will be a forest $F_{1}=\cup_{l=1}^{f_{1}} T_{1, l}$, with $T_{1, l} \subseteq E_{1}, l=1 \ldots f_{1}$. The subset of $F_{0}$ that remains valid for $G_{1}$ is denoted with $F_{0}^{\prime}$ and we refer with $T_{0, l}^{\prime}$ to the subset of the tree $T_{0, l}$ that remains valid in $G_{1}$. Thus it is

$F_{0}^{\prime}=\cup_{l=1}^{f_{0}} T_{0, l}^{\prime}$. Given two vertices $v_{i}$ and $v_{j}$ of some tree $T$, with $\left[v_{i} \cdots v_{j}\right]_{T}$ we denote the set of edges of the unique path connecting $v_{i}$ and $v_{j}$ on $T$.

\section{A repairing strategy}

In this paragraph we design and analyze probabilistically a repairing algorithm for an a priori feasible solution $F_{0}$. When the subgraph $G_{1}$ materializes, the algorithm identifies the trees of $F_{0}$ that become disconnected in $G_{1}$ (due to abscense of some edges incident to missing vertices), and reconnects each tree separately by using additional edges from $E_{1}$. Clearly this procedure generates a Steiner Forest that is feasible for the originally given pairs $\left\langle s_{r}, t_{r}\right\rangle$, $r=1 \ldots k$, and ensures $f_{1}=f_{0}$ i.e., both the a priori and repaired forests have the same number of trees. We explain the procedure followed by the algorithm for reconnecting a particular tree of $F_{0}$ that has been disconnected in $G_{1}$. This same procedure is followed for every such disconnected tree separately.

Consider the tree $T_{0, l}$, such that $T_{0, l}^{\prime} \subset T_{0, l}$. The algorithm orders the vertices of $T_{0, l}$ using a Depth-First-Search, starting from an arbitrary leaf-vertex of $T_{0, l}$. Vertices of $T_{0, l}$ are inserted in an ordered list $\mathcal{L}$ in order of visitation by DFS in the following way: if $v_{i}$ and $v_{i+1}$ are two distinct vertices visited by DFS consecutively for the first time, but no $\left(v_{i}, v_{i+1}\right)$ edge exists in $T_{0, l}$, then they are appended to $\mathcal{L}$ along with the parent vertex $u$ of $v_{i+1}$, in the order $v_{i}, u, v_{i+1}$. Thus $\mathcal{L}$ may contain some vertices more than once (in fact, as many times as their children in $\left.T_{0, l}\right)$. However, $|\mathcal{L}|=O\left(\left|T_{0, l}\right|\right)$. We note that a different ordered list is produced for each tree of the a priori solution that needs to be repaired.

When the actual subgraph $G_{1}$ materializes, the algorithm sets $T_{1, l}=T_{0, l}^{\prime}$. Then it removes from $\mathcal{L}$ every copy of vertex $v \in V_{0} \backslash V_{1}$ thus producing the list $\mathcal{L}^{\prime}$. It scans $\mathcal{L}^{\prime}$ in order and for every two consecutive vertices $v_{i}, v_{j}$ it inserts in $T_{1, l}$ an edge $\left(v_{i}, v_{j}\right)$ if $i<j$ and $v_{i}, v_{j}$ are not already connected in $T_{1, l}$. We illustrate the functionality of the repairing algorithm over a particular tree by an example.

Example Fig. 1(a) depicts a tree of an a priori solution numbered according to DFS visitation starting from a leaf-vertex. The corresponding ordered list produced in this way is $\mathcal{L}=\{1,2,3,4,2,5,6,2,7,8,7,9,10\}$. Assuming that vertices 2 and 7 are absent from the vertex set of the actually materialized subgraph, all occurences of these vertices are dropped from $\mathcal{L}$ and $\mathcal{L}^{\prime}=\{1,3,4,5,6,8,9,10\}$ emerges. The repairing agorithm scans $\mathcal{L}^{\prime}$ in order and adds edges $(1,3),(4,5),(6,8),(8,9)$, so as to reconnect the remainders of the a priori tree, as shown in fig. 1(b).

We prove the following:

Proposition 1 The repairing algorithm produces a connected tree $T_{1, l}$ out of tree $T_{0, l}$ of the a priori solution. 


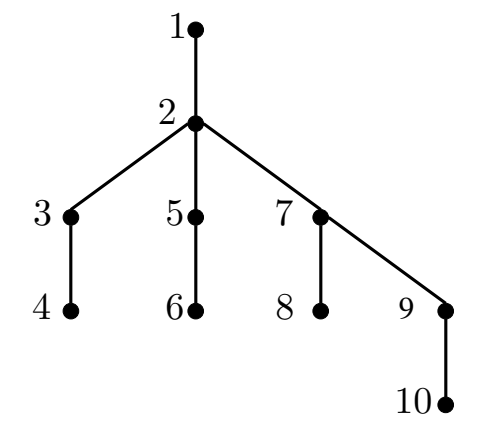

(a) A tree of the a priori solution.

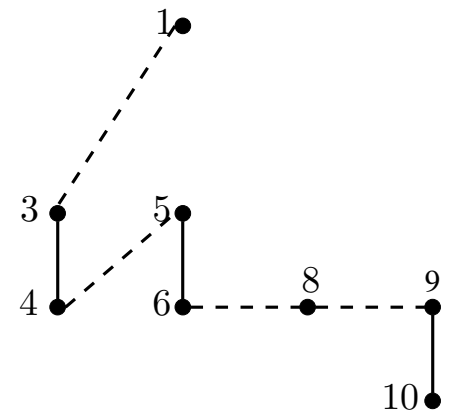

(b) The repaired tree in absence of vertices $v_{2}, v_{7}$. Dashed edges were added by the repairing algorithm.

Figure 1: Functionality of the repairing algorithm over a particular tree of an a priori forest.

Proof. For every vertex $v_{j}$ in $\mathcal{L}^{\prime}$ there is an appearance of $v_{j}$ in $\mathcal{L}^{\prime}$ after a vertex $v_{i}$ with $i<j$, so that $v_{j}$ is connected to $v_{i}$ by the end of the repairing algorithm. This holds for all vertices, apart from the one appearing first in $\mathcal{L}^{\prime}$. This implies that all vertices are connected into one component by the end of execution of the repairing algorithm for $T_{0, l}$. Furthermore the emerging construction cannot contain cycles for two reasons: $T_{0, l}$ did not have cycles and in order for a cycle to occur in the repaired solution $T_{1, l}$, insertion of at least one edge $\left(v_{i}, v_{j}\right)$ is required while its endpoints have been already connected. This cannot happen by functionality of the repairing algorithm.

Since the repairing algorithm reconnects on $G_{1}$ every single tree of the a priori solution that was disconnected, the union of all such repaired trees along with trees that survived unaffected on $G_{1}$ yields a feasible Steiner Forest on $G_{1}$. These trees remain pairwise vertexdisjoint as they were in the a priori solution, because the repairing algorithm uses only edges to reconnect trees and no such edge connects vertices belonging in different trees. Thus $f_{0}=f_{1}$ is guaranteed.

The complexity of the repairing algorithm is almost linear in the number of vertices of $G_{0}$. Indeed, a DFS over a tree $T_{0, l}$ is of $O\left(\left|T_{0, l}\right|\right)$ time, while by using UNION-FIND disjoint sets representation for maintaining connected components during the scan of $\mathcal{L}^{\prime}$, an $O\left(\left|T_{0, l}\right| \alpha\left(\left|T_{0, l}\right|\right)\right)$ time is spent. Summing over all trees of the a priori forest $F_{0}$, and because $\left|T_{0, l}\right|=O(n)$, we obtain $O(n \alpha(n))$ total time for producing the final feasible forest $F_{1}$.

Theorem 1 Given an arbitrary feasible a priori solution $F_{0}$, the expected cost of a repaired solution $F_{1}$ is:

$$
E\left[c\left(F_{1}\right)\right]=\sum_{l=1}^{f_{1}}\left(\sum_{\left(v_{i}, v_{j}\right) \in T_{0, l}} p_{i} p_{j} c\left(v_{i}, v_{j}\right)+\sum_{\left(v_{i}, v_{j}\right) \in E\left(V\left(T_{0, l}\right)\right) \backslash T_{0, l}} c\left(v_{i}, v_{j}\right) p_{i} p_{j} \times \prod_{\substack{v_{l} \in\left[v_{i}, v_{j}\right] \mathcal{L}_{l}: \\ i<j, v_{i}, v_{j} \notin\left[v_{i}, v_{j}\right] \mathcal{L}_{l}}}\left(1-p_{l}\right)\right)
$$

where $V\left(T_{0, l}\right)$ is the set of vertices incident to edges of $T_{0, l}$, and $E\left(V\left(T_{0, l}\right)\right)$ is the set of all edges induced by vertices in $V\left(T_{0, l}\right)$. Furthermore, $\mathcal{L}_{l}$ is the ordered list for tree $T_{0, l}$ and $\left[v_{i}, v_{j}\right]_{\mathcal{L}_{l}}$ the sublist of $\mathcal{L}_{l}$ starting at $v_{i}$ and ending in $v_{j}$ not including these two vertices. For all sublists not satisfying the specified restrictions we define the product to be 0 . 
Proof. Each individual expression summed for tree $T_{0, l}$ consists of two terms, the first one expressing the expected cost of surviving edges in the materialized subgraph (that is the expected cost of $T_{0, l}^{\prime}$ ), while the second expresses the expected cost of edges added to $T_{0, l}^{\prime}$ by the repairing algorithm, so that $T_{0, l}^{\prime}$ is augmented into a feasible tree $T_{1, l}$. The first term is justified by the fact that $\left(v_{i}, v_{j}\right) \in T_{0, l}$ survives in $T_{0, l}^{\prime}$ if and only if both its endpoints survive. This happens with probability $p_{i} p_{j}$, since these two events are independent.

The second term emerges by inspection of the functionality of the repairing algorithm. When $G_{1}$ materializes, missing vertices (in $V_{0} \backslash V_{1}$ ) are dropped from the ordered list encoding $\mathcal{L}_{l}$ and the modified list $\mathcal{L}_{l}^{\prime}$ emerges. The repairing algorithm scans $\mathcal{L}_{l}^{\prime}$ and for every pair of consecutive vertices $v_{i}, v_{j}$ it connects them using an edge $\left(v_{i}, v_{j}\right)$ if and only if $i<j$ and $v_{i}$ is not connected to $v_{j}$ already.

Vertices $v_{i}, v_{j} \in \mathcal{L}$ both survive in $\mathcal{L}_{l}^{\prime}$ with probability $p_{i} p_{j}$. Vertices $v_{i}$ and $v_{j}$ are not connected to each other if all vertices between $v_{i}$ and $v_{j}$ in $\mathcal{L}_{l}$ are missing from $\mathcal{L}_{l}^{\prime}$, and this happens with probability $\prod_{v_{l} \in\left[v_{i}, v_{j}\right]_{\mathcal{L}}}\left(1-p_{l}\right)$. Furthermore, neither $v_{i}$ nor $v_{j}$ should appear as intermediates in the sublist $\left[v_{i}, v_{j}\right] \mathcal{L}_{l}$, otherwise they should also be missing, and would not be encountered by the repairing algorithm. Finally, the sublist $\left[v_{i}, v_{j}\right]_{\mathcal{L}_{l}}$ should not be empty, otherwise a surviving edge $\left(v_{i}, v_{j}\right)$ is implied, rendering $v_{j}$ connected to $v_{i}$.

Clearly the expression given in theorem 1 is computable in polynomial-time. Thus:

Corrolary 1 The problem of a priori optimizing the steiner forest problem on stochastic metric graphs, for the proposed repairing algorithm, belongs to the class NPO.

The problem is $N P$-hard: setting all survival probabilities of vertices of $G_{0}$ equal to 1 , yields a deterministic steiner forest instance. Results of section 3 imply existence of a polynomial-time 4-approximation algorithm for a priori optimization of the expression of theorem 1.

\section{Approximation}

In this section we carry out appropriate analysis so as to show that the Steiner Forest problem over a stochastic metric graph can be approximated efficiently within a constant factor, by the repairing algorithm. The heart of our results is the following theorem:

Theorem 2 If $F_{1}$ is a repaired feasible solution produced by the proposed repairing algorithm for the Steiner Forest problem on a stochastic metric graph, given an a priori feasible solution $F_{0}$, then $c\left(F_{1}\right) \leq 2 c\left(F_{0}\right)$.

The proof of this result is carried out by an analysis of the algorithm over each tree $T_{0, l}$ separately. The result emerges by summing over the trees of $F_{1}$. In the following we denote by $T_{r, l}$ the subset of edges added by the repairing algorithm to $T_{0, l}^{\prime}$. We prove first some lemmas that will be combined towards the proof of the theorem.

Lemma 1 For every edge $\left(v_{i}, v_{j}\right) \in T_{r, l}$ we have $c\left(v_{i}, v_{j}\right) \leq c\left(\left[v_{i} \ldots v_{j}\right]_{T_{0, l}}\right)$.

Proof. Immediate by the triangle inequality holding for the cost function $c: E_{0} \rightarrow \Re^{+}$.

According to lemma 1 we can express the cost of the repaired tree $T_{1, l}$ as follows:

$$
c\left(T_{1, l}\right)=c\left(T_{0, l}^{\prime}\right)+c\left(T_{r, l}\right) \leq \sum_{e \in T_{0, l}^{\prime}} c(e)+\sum_{\left(v_{i}, v_{j}\right) \in T_{r, l}} c\left(\left[v_{i} \ldots v_{j}\right]_{T_{0, l}}\right)
$$



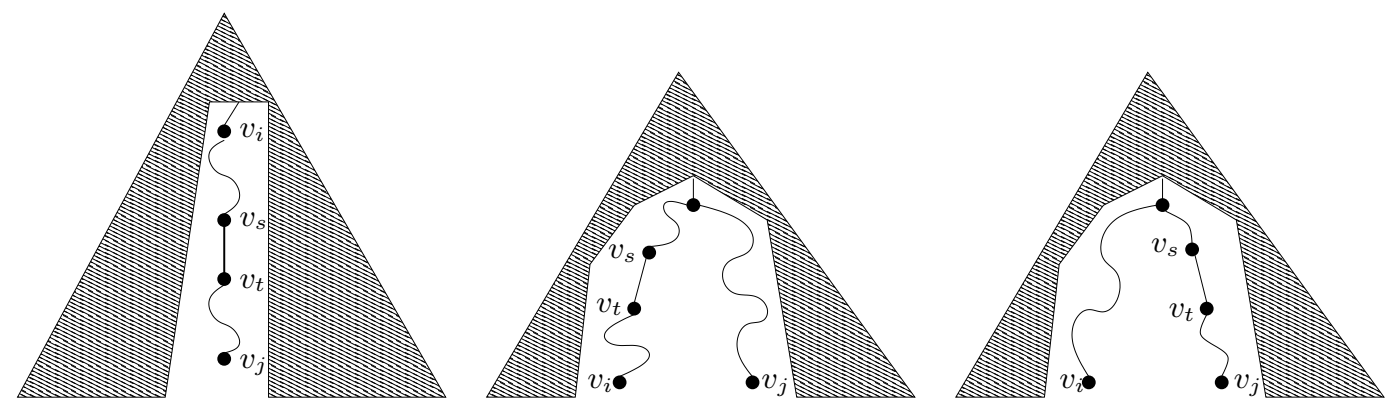

Figure 2: Three cases that may happen for edge $\left(v_{s}, v_{t}\right)$ with respect to $v_{i}, v_{j}$ (proof of lemma 2).

Lemma 2 For every three distinct edges $\left(v_{i}, v_{j}\right),\left(v_{k}, v_{l}\right),\left(v_{q}, v_{r}\right)$ in $T_{r, l}$ the paths $\left[v_{i} \cdots v_{j}\right] T_{0, l}$, $\left[v_{k} \cdots v_{l}\right]_{T_{0, l}},\left[v_{q} \cdots v_{r}\right]_{T_{0, l}}$, do not share an edge in common.

Proof. By functionality of the repairing algorithm we have that $i<j, k<l, q<r$. Furthermore, if we assume without loss of generality that the vertex pairs were encountered in the order $\langle i, j\rangle,\langle k, l\rangle,\langle q, r\rangle$ during scanning of $\mathcal{L}^{\prime}$, then we deduce that $j<l<r$. If the paths intersect in some common edge $\left(v_{s}, v_{t}\right)$, then it must be $s, t \leq j$ (fig. 2 depicts all possible cases), thus $s, t<l$ and $s, t<r$. In this case edge $\left(v_{s}, v_{t}\right)$ must have been scanned at least three times during DFS: once before visitation of each of the vertices $v_{j}, v_{l}, v_{r}$. But this contradicts the fact that a DFS scans each edge of a graph exactly twice.

The following lemma will help us to complete the proof of the theorem:

Lemma 3 Consider two edges $\left(v_{i}, v_{j}\right),\left(v_{k}, v_{l}\right)$ in $T_{r, l}$. For every edge $\left(v_{s}, v_{t}\right)$ with $\left(v_{s}, v_{t}\right) \in$ $\left[v_{i} \cdots v_{j}\right]_{T_{0, l}} \cap\left[v_{k} \cdots v_{l}\right]_{T_{0, l}}$ it holds $\left(v_{s}, v_{t}\right) \notin T_{0, l}^{\prime}$.

Proof. The proof is by contradiction. Suppose that $\left(v_{s}, v_{t}\right) \in\left[v_{i} \cdots v_{j}\right]_{T_{0, l}} \cap\left[v_{k} \cdots v_{l}\right]_{T_{0, l}}$ and $\left(v_{s}, v_{t}\right) \in T_{0, l}^{\prime}$. Without loss of generality we assume that the repairing algorithm encountered first the pair $\left\langle v_{i}, v_{j}\right\rangle$ and afterwards the pair $\left\langle v_{k}, v_{l}\right\rangle$ in $\mathcal{L}^{\prime}$. It must be $i<j, k<l$ and $j<l$ $\left(v_{j}\right.$ may coincide with $\left.v_{k}\right)$. Since $\left(v_{s}, v_{t}\right) \in\left[v_{i} \cdots v_{j}\right]_{T_{0, l}} \cap\left[v_{k} \cdots v_{l}\right]_{T_{0, l}}$, then we must have $s, t \leq j$ and, consequently, $s, t<l$. Furthermore, it must hold either that (i) $s, t>k$ or that (ii) $s, t>i$, otherwise it should be $s, t<i$ and, given that $s, t \leq j$ also, we would deduce that $\left(v_{s}, v_{t}\right)$ would have been scanned twice during DFS, once before visitation of $v_{i}$ and once before visitation of $v_{j}$. In this case it could not have been scanned again right before visitation of $v_{l}$. Now (i) cannot hold because $k \geq j$ and $s, t<j$. If (ii) holds, i.e. $s, t>i$, it is implied that the repairing algorithm did not encounter in $\mathcal{L}^{\prime}$ vertices $v_{k}, v_{l}$ and $v_{i}, v_{j}$ consecutively (fig. 3), which is a contradiction.

The proof of theorem 2 can now be completed as follows:

Proof. Relation (1) can be written:

$$
\begin{gathered}
c\left(T_{1, l}\right) \leq \sum_{e \in T_{0, l}^{\prime}} c(e)+\sum_{\left(v_{i}, v_{j}\right) \in T_{r, l}} c\left(\left[v_{i} \ldots v_{j}\right]_{T_{0, l}}\right)=\sum_{e \in T_{0, l}^{\prime}} c(e)+\sum_{\left(v_{i}, v_{j}\right) \in T_{r, l}} \sum_{e \in\left[v_{i} \ldots v_{j}\right]_{T_{0}, l}} c(e) \Rightarrow \\
c\left(T_{1, l}\right) \leq \sum_{e \in T_{0, l}^{\prime}} c(e)+\sum_{\left(v_{i}, v_{j}\right) \in T_{r, l}}\left(\sum_{e \in\left[v_{i} \ldots v_{j}\right]_{T_{0}, l}: e \in T_{0, l}^{\prime}} c(e)+\sum_{e \in\left[v_{i} \ldots v_{j}\right]_{T_{0, l}}: e \notin T_{0, l}^{\prime}} c(e)\right)
\end{gathered}
$$




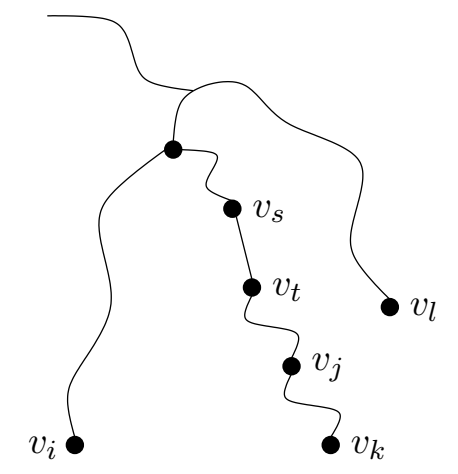

Figure 3: Case $s, t>i$ examined in the proof of lemma 3: $\left(v_{s}, v_{t}\right) \in\left[v_{i} \cdots v_{j}\right]_{T_{0, l}} \cap\left[v_{k} \cdots v_{l}\right]_{T_{0, l}}$ and suppose that $\left(v_{s}, v_{t}\right) \in T_{0, l}^{\prime}$. Then obviously $v_{s}, v_{t}$ survive in $\mathcal{L}^{\prime}$ and appear as intermediates in the two pairs $\left\langle v_{i}, v_{j}\right\rangle$ and $\left\langle v_{k}, v_{l}\right\rangle$.

By lemmas 2 and 3 the following are implied:

$$
\begin{gathered}
\sum_{\left(v_{i}, v_{j}\right) \in T_{r, l}} \sum_{e \in\left[v_{i} \ldots v_{j}\right]_{T_{0, l}}: e \in T_{0, l}^{\prime}} c(e) \leq \sum_{e \in T_{0, l}^{\prime}} c(e) \\
\sum_{\left(v_{i}, v_{j}\right) \in T_{r, l}} \sum_{e \in\left[v_{i} \ldots v_{j}\right]_{T_{0, l}}: e \notin T_{0, l}^{\prime}} c(e) \leq 2 \sum_{e \in T_{0, l} \backslash T_{0, l}^{\prime}} c(e)
\end{gathered}
$$

By replacing the relations (3) and (4) in the expression (2) we obtain:

$$
c\left(T_{1, l}\right) \leq 2 \sum_{e \in T_{0, l}^{\prime}} c(e)+2 \sum_{e \in T_{0, l} \backslash T_{0, l}^{\prime}} c(e) \leq 2 c\left(T_{0, l}\right)
$$

Summing over all trees, since $f_{0}=f_{1}$, we obtain that $c\left(F_{1}\right) \leq 2 c\left(F_{0}\right)$.

Now we can state our main approximation result:

Theorem 3 There is an almost linear time repairing algorithm for Steiner Forest problem on stochastic metric graphs that, when applied to an $\alpha$-approximate a priori feasible solution, produces feasible solutions that are $2 \alpha$-approximate to the optimum expected cost.

Proof. By theorem $2 c\left(F_{1}\right) \leq 2 c\left(F_{0}\right)$. Let $O P T\left(G_{0}\right)$ and $O P T\left(G_{1}\right)$ be the costs of an optimum Steiner forest on $G_{0}$ and $G_{1}$ respectively for the given source-destination pairs, and $c\left(F_{0}\right) \leq \alpha O P T\left(G_{0}\right)$. It is $\operatorname{OPT}\left(G_{0}\right) \leq O P T\left(G_{1}\right)$ for every possible subgraph $G_{1}$ of $G_{0}$, because every feasible solution for $G_{1}$ is also feasible for $G_{0}$. Thus $c\left(F_{1}\right) \leq 2 \alpha O P T\left(G_{1}\right)$. Taking expectation over all possible subgraphs $G_{1}$ yields $E\left[c\left(F_{1}\right)\right] \leq 2 \alpha E\left[O P T\left(G_{1}\right)\right]$.

Corrolary 2 There is an $O(n \alpha(n))$ time repairing algorithm for the Steiner Forest problem on stochastic metric graphs, that can be supported by a polynomial-time algorithm for taking a priori decisions [1,8], so as to yield factor 4 approximation of the optimum expected cost. The repairing algorithm is 2-approximate given an a priori optimum feasible solution.

We note that in both cases mentioned in the corollary, the proposed repairing algorithm is faster than the algorithm used for a priori decisions, and is far more efficient than the trivial practices discussed in the introduction: in fact, any approximation algorithm used for taking a priori decisions (including the one of $[1,8]$ ) will incur $\Omega\left(n^{2}\right)$ complexity. 


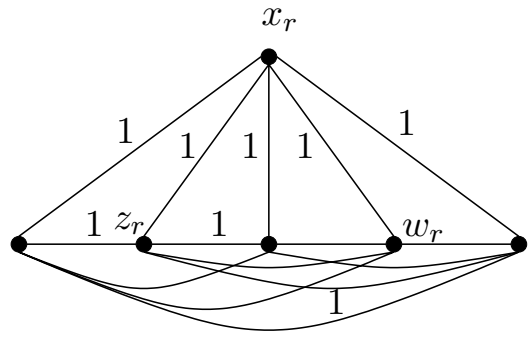

(a) The structure used in proving tightness of analysis for some $S_{r}$, with $\left|S_{r}\right|=$ 5. Apart from edges with cost 1 , all other edges have cost 2 .

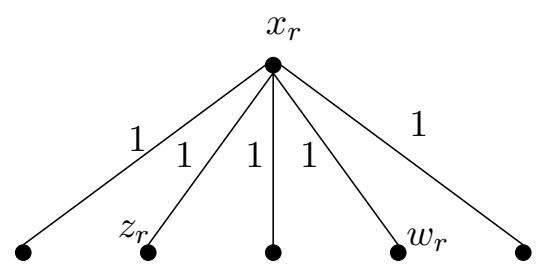

(b) An optimum star centered at $x_{r}$, with cost 5.

Figure 4: Illustration of worst-case construction for showing tightness of analysis.

\section{Tightness of Analysis}

We show in this paragraph that the analysis of the repairing algorithm is tight for arbitrary a priori solution $F_{0}$. We construct a worst-case example.

We consider a metric graph $G_{0}\left(V_{0}, E_{0}\right)$. For some fixed constant $k$ take $k$ sets of vertices $S_{1}, \ldots, S_{k}$, along with a vertex $x_{r} \notin S_{r}, r=1 \ldots k$, per subset. Let the input metric graph consist of the vertex set $V_{0}=\left(\cup_{r=1}^{k} S_{r}\right) \cup\left\{x_{r} \mid r=1 \ldots k\right\}$. We take $\left|S_{r}\right|=n$ so that $\left|V_{0}\right|=\Theta(n)$, because $k$ is a fixed constant. We set $c\left(x_{r}, y\right)=1$ for each $y \in S_{r}, r=1 \ldots k$. For each $S_{r}$ pick two distinct arbitrary vertices $w_{r}, z_{r} \in S_{r}$ and set $c\left(z_{r}, y\right)=1$ for all $y \in S_{r} \backslash\left\{w_{r}, z_{r}\right\}$ and $c\left(z_{r}, w_{r}\right)=2$. For all other edges of the graph we set their cost equal to 2. Fig. 4(a) shows the construction for a particular set $S_{r}$.

The Steiner Forest instance that we consider requires that each $S_{r}$ is connected (it is trivial to express this requirement with source-destination pairs). We assume that the stochastic graph is defined by setting the survival probability of each $x_{r}$ equal to $p$. An optimum a priori solution to this instance is defined as a forest consisting of an optimum connecting tree per vertex set $S_{r}$. We consider such an a priori solution that the corresponding tree for $S_{r}$ is the star $T_{r}=\left\{(x, y) \mid y \in S_{r}\right\}$. Fig. 4(b) shows the construction for a particular vertex set $S_{r}$ and the optimum star tree solution for this set.

Among the various cases that may occur in the actually materialized subgraph of $G_{0}$ we consider the one where all vertices $x_{r}, r=1 \ldots k$ survive, and the case where all vertices $x_{r}$ are missing. For the first case the a priori optimum solution remains feasible and has an optimum cost of $\sum_{r=1}^{k}\left|S_{r}\right|$, while in the second case, the repairing algorithm is executed for each tree $T_{r}$. Fig. 5 depicts the DFS numbering of a tree $T_{r}$ by the repairing algorithm, and the corresponding repaired solution. It is easy to see that such a "chain" as the one appearing in fig. 5(b), must have a cost at least $2\left(\left|S_{r}\right|-1\right)-2=2\left(\left|S_{r}\right|-2\right)$, because in this chain $z_{r}$ may be incident to two vertices that are connected with two edges of cost 1 to it. However, the optimum cost for the materialized subgraph occurs if we connect per set $S_{r}$ its vertices to $z_{r}$, and is equal to $\left|S_{r}\right|$. Clearly the optimum expected cost is at most $\sum_{r=1}^{k}\left|S_{r}\right|=k(n-1)$, while the solution produced by the repairing algorithm has an expected cost of value at least $p^{k} \sum_{r=1}^{k}\left|S_{r}\right|+2\left(1-p^{k}\right) \sum_{r=1}^{k}\left(\left|S_{r}\right|-2\right)=k p^{k}(n-1)+2 k\left(1-p^{k}\right)(n-2)$. Hence, the approximation factor is asymptotically lower-bounded by: $\lim _{n \rightarrow \infty} \frac{k p^{k}(n-1)+2 k\left(1-p^{k}\right)(n-2)}{k(n-1)}=$ $p^{k}+2\left(1-p^{k}\right)$, which approaches arbitrarily close to 2 by choosing $p$ arbitrarily close to 0 . 


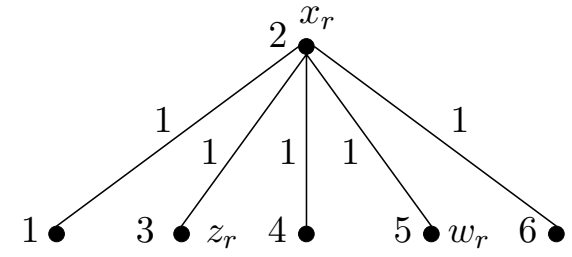

(a) DFS numbering produced by the repairing algorithm for star connection of $S_{r}$.

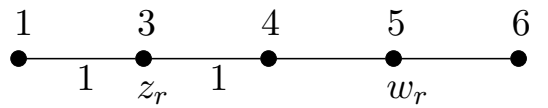

(b) A feasible tree produced over a disconnected star tree, when $x_{r}$ is missing.

Figure 5: DFS numbering and repaired tree assumed in showing tightness of analysis.

\section{Conclusions}

We considered the Steiner Forest problem in stochastic metric graphs, where each vertex that is not a source or destination is present with a given probability independently of all other vertices. The problem amounts to coming up with a feasible Steiner Forest for every possible materializable subgraph of the given graph, so as to minimize the expected cost of the resulting solution taken over the distribution of these subgraphs. We designed an efficient algorithm that runs in almost linear time in the number of vertices that adjusts efficiently a priori taken decisions. Given that a priori decisions constitute a feasible forest on the original metric graph we were able to derive a polynomial time computable expression for the expected cost of a Steiner Forest produced by the proposed algorithm. Furthermore, we have shown that this algorithm at most doubles the cost of the a priori solution, and this leads to 2 approximation of the optimum expected cost given an optimum a priori solution, and 4 approximation given a 2 approximate solution. Our analysis of the proposed repairing algorithm was shown to be tight.

We note that for the more special case of the Steiner Tree problem in the same model, the well-known minimum spanning tree heuristic [18] that includes only vertices requiring connection, gives a feasible and 2-approximate a priori solution that trivially remains feasible and 2-approximate for the actually materialized subgraph. As a non-trivial aspect of future work we consider extending our results to the case of complete graphs with general cost functions. Simply using shortest-path distances on these graphs does not straightforwardly lead to efficient and approximate repairing algorithms.

\section{References}

[1] A. Agrawal, P. N. Klein, and R. Ravi. When Trees Collide: An Approximation Algorithm for the Generalized Steiner Problem on Networks. SIAM Journal on Computing, 24(3):440-456, 1995.

[2] D. Bertsimas. Probabilistic Combinatorial Optimization. PhD thesis, 1988.

[3] D. Bertsimas. The probabilistic minimum spanning tree problem. Networks, 20:245-275, 1990.

[4] J. R. Birge and F. Louveaux. Introduction to Stochastic Programming. Springer-Verlag New York, 1997. 
[5] G. W. Dantzig. Linear programming under uncertainty. Management Science, 1:197-206, 1951.

[6] K. Dhamdhere, R. Ravi, and M. Singh. On Two-Stage Stochastic Minimum Spanning Trees. In Proceedings of the International Conference on Integer Programming and Combinatorial Optimization (IPCO), pages 321-334, 2005.

[7] L. Fleischer, J. Koenemann, S. Leonardi, and G. Schaefer. Simple cost sharing schemes for multicommodity rent-or-buy and stochastic steiner tree. In Proceedings of the ACM Symposium on Theory of Computing (STOC), pages 663-670, 2006.

[8] M. X. Goemans and D. P. Williamson. A General Approximation Technique for Constrained Forest Problems. SIAM Journal on Computing, 24(2):296-317, 1995.

[9] A. Gupta and M. Pál. Stochastic Steiner Trees Without a Root. In Proceedings of the International Colloquium on Automata, Languages and Programming (ICALP), pages 1051-1063, 2005.

[10] A. Gupta, M. Pál, R. Ravi, and A. Sinha. What About Wednesday? Approximation Algorithms for Multistage Stochastic Optimization. In Proceedings of the International Workshop on Approximation and Randomized Algorithms (APPROX-RANDOM), pages 86-98, 2005.

[11] A. Gupta, Martin Pál, R. Ravi, and A. Sinha. Boosted sampling: approximation algorithms for stochastic optimization. In Proceedings of the ACM Symposium on Theory of Computing (STOC), pages 417-426, 2004.

[12] A. Gupta, R. Ravi, and A. Sinha. An Edge in Time Saves Nine: LP Rounding Approximation Algorithms for Stochastic Network Design. In Proceedings of the IEEE Symposium on Foundations of Computer Science (FOCS), pages 218-227, 2004.

[13] N. Immorlica, D. R. Karger, M. Minkoff, and V. S. Mirrokni. On the costs and benefits of procrastination: approximation algorithms for stochastic combinatorial optimization problems. In Proceedings of the ACM-SIAM Symposium on Discrete Algorithms (SODA), pages 691-700, 2004.

[14] P. Jaillet. Probabilistic Traveling Salesman Problems. PhD thesis, 1985.

[15] C. Murat and V. Th. Paschos. The probabilistic longest path problem. Networks, 33(3):207-219, 1999.

[16] C. Murat and V. Th. Paschos. A priori optimization for the probabilistic maximum independent set problem. Theoretical Computer Science, 270(1-2):561-590, 2002.

[17] C. Murat and V. Th. Paschos. On the probabilistic minimum coloring and minimum k-coloring. Discrete Applied Mathematics, 154(3):564-586, 2006.

[18] V. Vazirani. Approximation Algorithms. Springer-Verlag, Heidelberg, 2003. 